\section{Evidencia serológica retrospectiva de infecciones por Leptospira spp., dengue, hantavirus y arenavirus en indígenas Emberá-Katío, Colombia}

\author{
Berta Restrepo, Juan D. Rodas, Carolina Montoya-Ruiz, \\ Angélica M. Zuluaga, Gabriel Parra-Henao y Piedad Agudelo-Flórez
}

Serological evidence of Leptospira spp., dengue, hantavirus and arenavirus infection in Embera-Katío indigenous population, Colombia

We have developed a study in the Embera-Katío indigenous community and we found that the seropositivity for IgG antibodies to Leptospira spp. was $18.1 \%$; to dengue was $61.1 \%$; to arenavirus was $3.1 \%$ and to hantavirus was $1.5 \%$. We conclude that this indigenous community live in conditions that promote the reemerging and emerging, infections

Key words: Leptospirosis; dengue; arenavirus; hantavirus; indigenous population; serology.

Palabras clave: Leptospirosis; dengue; arenavirus; hantavirus; población indígena, serología.

\section{Introducción}

La comunidad indígena Embera-Katío se ha establecido históricamente en el Alto Sinú (Córdoba, Colombia), una región selvática con condiciones de pobreza, aislamiento y conflicto armado ${ }^{1}$. El objetivo de este estudio fue investigar, en forma retrospectiva, la seroprevalencia de infecciones por Leptospira spp, virus del dengue, hantavirus y arenavirus, y la asociación entre la seropositividad a estos agentes y variables socio-demográficas.

\section{Método}

Se evaluaron muestras de sueros disponibles en el Instituto Colombiano de Medicina Tropical-Universidad CES, obtenidas previamente en un estudio transversal realizado entre noviembre de 2001 y mayo de 2002

Instituto Colombiano de Medicina Tropical-Universidad CES, Antioquia, Colombia (BR) Grupo de Epidemiología y Bioestadística (GPH).

Facultad de Medicina, Grupo de Ciencias Básicas (PAF).

Universidad de Antioquia.

Grupo Centauro (JDR, CMR, AMZ).

Los autores declaramos no tener conflictos de interés.

El proyecto fue financiado por el Hospital San José, Córdoba, Colombia, por el Instituto Colombiano de Medicina Tropical, y recursos de la estrategia de sostenibilidad 2013-2014 de la Vicerrectoria de Investigación de la Universidad de Antioquia para el grupo Centauro. Las pruebas de ELISA para Leptospira fueron donadas por el Dr. Manuel Céspedes del Instituto Nacional de Salud de Perú.

Recibido: 9 de diciembre de 2015 / Aceptado: 4 de julio de 2016

\section{Correspondencia a:}

Berta Restrepo

brestrepo@ces.edu.co en el Resguardo Embera-Katio. La muestra se calculó con una precisión de $5 \%$, un nivel de confianza de $95 \%$ y una prevalencia de $50 \%$, para un total de 333 individuos, que se amplió en $20 \%$ por posibles pérdidas. La selección de la muestra fue por muestreo aleatorio simple. La detección de anticuerpos IgG para Leptospira spp. fue realizada con la metodología descrita por Céspedes y cols ${ }^{2}$. A las muestras positivas se les determinó el serogrupo por microaglutinación (MAT) según la metodología del Instituto Nacional de Salud de Perú2,3. La detección de IgG para dengue se realizó con UMELISA ${ }^{\circledR}$ IgG Dengue (Centro de Inmunoensayo, Instituto Pedro Kouri de la Habana, Cuba) y para IgM se utilizó Panbio ${ }^{\circledR}$ (Alere, Sinnamon Park, Australia). La detección de IgG para hantavirus y arenavirus se realizó por ELISA, usando lisados como antígenos de células Vero infectadas con el hantavirus Maciel o con el arenavirus Junín (Instituto Nacional de Enfermedades Virales Humanas-INEVH). Se calculó el índice de depósito de A. aegypti en cuatro comunidades (selváticas) y en el barrio Esmeralda (urbano) según metodología descrita ${ }^{4,5}$.

La seropositividad para anticuerpos IgG a Leptospira spp. fue 18,1\% (71/391), dengue: $61,1 \%(258 / 422)$, arenavirus: $3,1 \%(10 / 325)$, hantavirus: $1,5 \%(5 / 324)$ e IgM para dengue fue $5 \%(21 / 391)$. El serogrupo más frecuente de Leptospira fue Australis Bratislava, seguido por Icterohaemorrhagiae y Panamá. El incremento de seropositividad para IgG a Leptospira spp. y dengue estuvo asociada a mayor edad $(\mathrm{p}>0,001)$. Se observó asociación entre la ocupación de agricultor y la seropositividad a IgG para Leptospira spp. vs. no tener ocupación $(32,9 \%$ vs $13 \%$; OR $=3,27$; IC95\% $=1,13-9,89)$ y entre ser estudiante y seropositividad a IgG para dengue ( $80,8 \%$ vs. $46 \%$; $\mathrm{OR}=4,93$, IC95\% = 2,09-11,8). No se observó $A$. aegypti en las cuatro comunidades ubicadas a lo largo del Río Sinú, pero sí en el barrio Esmeralda donde las nueve llantas estudiadas tenían presencia de larvas de A. aegypti.

\section{Discusión}

Este estudio evidenció la circulación de cuatro agentes infecciosos emergentes y re-emergentes, en la población indígena Embera-Katío, los que se suman a otros problemas de salud de esta comunidad ${ }^{1}$. La seropositividad a estos agentes fue similar a la observada en regiones cercanas ${ }^{6-9}$. La presencia de serovariedades de Leptospira spp. de origen silvestre, como Australis Bratislava y Panamá ${ }^{10}$, evidencia la contaminación con orina de suelos y agua de reservorios silvestres, frecuentes en el ambiente selvático. Además, se confirmó la circulación urbana y no rural de $A$. aegypti en esa región. Esta información reviste interés para las autoridades indígenas, pues no se disponía información previa. Sin embargo, es necesario realizar nuevos estudios para actualizar la situación epidemiológica de agentes infecciosos en esta comunidad.

Agradecimientos: A la comunidad Embera-Katio del Alto Sinú Tierralta, Córdoba y a Dra. Silvana Levis del Instituto Nacional de Enfermedades Virales Humanas “Dr. Julio I. Maiztegui”, Pergamino, Argentina.

\section{Referencias bibliográficas}

1. Restrepo B N, Restrepo M T, Beltrán J C, Rodríguez M, Ramírez R E. Nutritional status of indigenous children aged up to six years in the Embera-Katio Indian reserve in Tierralta, Cordoba, Colombia. Biomedica 2006; 26: 517-27. 
2. Céspedes M, Glenny M, Felices V, Balda L, Suárez V. Prueba de ELISA indirecta para la detección de anticuerpos IgM para el diagnóstico de leptospirosis humana. Rev Peru Med Exp Salud Publica 2002; 19: 24-7.

3. Ministerio de Salud del Perú. Instituto Nacional de Salud. Manual de procedimientos bacteriológico y serológico para el diagnóstico de leptospirosis. 2002. Disponible en: http://bvs.minsa.gob.pe/local/ minsa/1049_INS-NT34.pdf (Fecha de acceso: 10 de marzo de 2013)

4. World Health Organization. Special Programme for Research and Training in Tropical Diseases (TDR). Dengue guidelines for diagnosis, treatment, prevention and control, 2009. Disponible en: http://www.who.int/tdr/ publications/documents/dengue-diagnosis.pdf

5. World Health Organization, Regional Office for the Western Pacific (WPRO), Guidelines for dengue surveillance and mosquito control, Western Pacific education in action series $\mathrm{N}^{\circ} 8$, World Health Organization, Manila, Philippines, 1995. Disponible en: http://apps.who.int/iris/ bitstream/10665/207027/1/9290611383_eng.pdf

6. Londoño A F, Díaz F J, Agudelo-Flórez P, Levis S, Rodas J D. Genetic evidence of hantavirus infections in wild rodents from northwestern Colombia. Vector Borne Zoonotic Dis 2011; 11: 701-8.

7. Arroyave E, Londoño A F, Quintero J C, Agudelo-Flórez P, Arboleda M, Díaz F J, et al. Etiology and epidemiological characterization of non-malarial febrile syndrome in three municipalities of Urabá (Antioquia), Colombia. Biomedica 2013; 33 Suppl 1: 99-107.

8. Agudelo-Flórez P, Restrepo-Jaramillo B N, Arboleda-Naranjo M. Leptospirosis in Uraba, Antioquia, Colombia: a seroepidemiological and risk factor survey in the urban population. Cad Saude Pública 2007; 23 : 2094-102.

9. Restrepo BN, Arboleda M, Lopera T. Estudio seroepidemiológico de dengue en la región del Urabá Antioqueño, Colombia. Infectio 2004; 8: 255-62 .

10. Brenner D J, Kaufmann A F, Sulzer K R, Steigerwalt A G, Rogers F C, Weyant R S. Further determination of DNA relatedness between serogroups and serovars in the family Leptospiraceae with a proposal for Leptospira alexanderi sp. nov. and four new Leptospira genomospecies. Int J Syst Bacteriol 1999; 49 Pt 2: 839-58. 